\title{
Position dependent mass Scarf Hamiltonians generated via the Riccati equation
}

\author{
S Cruz y Cruz@ | C Santiago-Cruz
}

Instituto Politécnico Nacional, UPIITA, Av. Instituto Politécnico Nacional 2580, C.P. 07340 Ciudad de México, Mexico

\section{Correspondence}

S Cruz y Cruz, Instituto Politécnico

Nacional, UPIITA, Av. Instituto

Politécnico Nacional 2580, C.P. 07340

Ciudad de México, Mexico.

Email: sara.cruzycruz@gmail.com

Communicated by: R. Porter

\section{Funding information}

CONACyT, Grant/Award Number: 408518; Instituto Politécnico Nacional, México, Grant/Award Number: 20180377; Spanish MINECO, Grant/Award Number: MTM2014-57129-C2-1-P; Junta de Castilla y León, Grant/Award Number: VA057U16

MSC Classification: 81Q10; 81Q60; 81R15
The construction of position dependent mass Scarf Hamiltonians of the trigonometric as well as the hyperbolic types is addressed by means of the factorization method and the Riccati equation. These Hamiltonians are shown to be independent of the ordering parameter of the kinetic term. Additionally, new families of Hamiltonians with the Scarf spectrum are also determined by supersymmetry. Some examples for masses with and without singularities are considered to illustrate our results.

\section{KEYWORDS}

Position-dependent mass, Scarf potentials, Special quantum systems, such as solvable systems, Supersymmetry and quantum mechanics

\section{1 | INTRODUCTION}

One of the most studied problems in quantum mechanics is the generation of exactly solvable models. In this context, the factorization or intertwining technique has played a very important role $\mathrm{e}^{1-3}$. In this method, a family of second order differential Hamiltonians, called Hamiltonian hierarchy, is expressed, up to an additive constant, as the product of a pair of first order operators. The factorization leads to a set of intertwining relations, linking different Hamiltonians in the hierarchy, from which the discrete spectrum, as well as the corresponding eigenfunctions can be algebraically determined for the entire hierarchy. Furthermore, the factorizing operators constitute the basis to determine the so called potential and/or spectrum generating algebras ${ }^{4}$ that may be used to construct the group theoretical approach of the corresponding model. From the inverse techniques point of view, the factorization method has found many applications in the generation of exactly solvable potentials with specific spectral properties. Those include shape-invariant and supersymmetric potentials..$^{5-10}$ Of particular interest is the generation of exactly solvable Hamiltonians endowed with position dependent mass (PDM) due to the fact that they have multiple applications in many areas of physics such as the description of optical and electronic properties of semiconductors and quantum dots, ${ }^{11-14}$ the construction of mechanical models in spaces with curvature, ${ }^{15,16}$ and the study of galactic mass loss ${ }^{17}$ among others. The use of the factorization method in considering PDM Hamiltonians has deserved growing attention in the last decades. ${ }^{18-29}$ Group theoretical approaches and shape-invariance techniques have been used in order to generate exactly solvable models. ${ }^{21,26,30,31}$ The problem of constructing PDM Hamiltonians with specific spectral properties is of special interest as it may have applications in modeling low dimensional structures as quantum dots and quantum wells. In this case, the spectrum of the system is defined, and the corresponding interaction potential must be determined for an arbitrary choice of the mass. Some contributions in this context include the use of the intertwining technique for the reconstruction of the well potential from its spectrum, ${ }^{32}$ and the combina- 
tion of the shape-invariance technique with the point canonical transformation to fix the spectrum of the system and to map the problem to a constant mass Schrödinger one in order to find the solution ${ }^{26}$.

As it is well known, the factorization method is closely related to the Riccati equation. ${ }^{33}$ Indeed, if a constant mass Hamiltonian $H=-\partial_{x x}+V$ is factorizable in the form $H=a^{+} a^{-}+\epsilon$ with $a^{+}=-g^{-1} \partial_{x} g, a^{-}=\left(a^{+}\right)^{\dagger}$ and $g$ a real-valued function, then $g$ is given by $g=\exp \left\{\int^{x} w(y) d y\right\}$, where $w$ is a solution of the Riccati equation $w_{x}+w^{2}+$ $\epsilon=V$. The algebraic properties of the operators $a^{ \pm}$encode the spectral information of the Hamiltonian $H$. The Riccati equation, thus, establishes a connection between the interaction potential and the corresponding spectrum generating algebra underlying the system. In the PDM case, this connection can be as well established, meaning that it is possible the construction of PDM Hamiltonians with a prescribed potential or spectrum generating algebra by means of the solutions of the appropriate Riccati equation.

In this work, we make use of the Riccati equation to construct PDM Hamiltonian families of the trigonometric as well as hyperbolic Scarf types (Scarf I and II types, respectively). Departing from a general PDM Hamiltonian hierarchy with a positive definite, but otherwise arbitrary mass function, we directly apply the factorization method to fix the underlying potential algebra as that of the Scarf type. The resulting Riccati equations are used to determine the corresponding family of PDM Scarf potentials, and their corresponding eigenfunctions are constructed by means of the intertwining relations. Our results are consistent with that of the point canonical transformation scheme. In the framework of PDM systems, an important concern corresponds to the choice of a particular ordering in the kinetic term of the Hamiltonian due to the noncommutativity of the mass $m(x)$ and the linear momentum $P . .^{24,30,34-36} \mathrm{In}$ our approach, the problem is addressed without any choice of the ordering label, and it is shown that the final result is, indeed, independent of the value of this parameter.

Our work is organized as follows. In Section 2, the PDM Schrödinger problem is considered in the framework of the factorization method. Section 3 contains the construction of trigonometric and hyperbolic Scarf type PDM potentials, and the underlying potential algebras are briefly discussed. In Section 4 these families of Scarf potentials are used as the seeds to constructing new Darboux-deformed potentials with the Scarf spectrum. Finally, we summarize our results in Section 5.

\section{2 | THE PDM SCHRÖDINGER PROBLEM}

In this section, we consider the PDM Schrödinger problem with the Hamiltonian

$$
H=m^{a} P m^{2 b} P m^{a}+V=T_{a}+V, \quad a+b=-\frac{1}{2},
$$

where the mass $m$ is a given, positive definite, real-valued function of the position, the labels $a, b$ are 2 parameters indicating the ambiguity in the ordering of the mass $m(x)$ and the linear momentum $P$ in the kinetic term, and $V$ is the potential that must be determined in such a way that $H$ fulfills some specific spectral properties. The domain of definition of $H$, $\operatorname{Dom}(H)$, will be fixed by the domain of definition of the mass $\operatorname{Dom}(m)$ and the potential $\operatorname{Dom}(V)$.

To address this problem, let us assume that the Hamiltonian can be factorized in terms of 2 linear operators $A, B$ as follows:

$$
H=A B+\epsilon,
$$

where

$$
A=-i m^{a} P m^{b}+W, \quad B=(A)^{\dagger}=i m^{b} P m^{a}+W,
$$

with $W$ a real-valued function of the position to be determined and $\epsilon$ a constant to be fixed.

The linear momentum fulfills the commutation relation $[X, P]=i$, with $X$ the position operator. In the position representation $X=x, P=-i \frac{d}{d x}$ and $[f(x), P]=i \frac{d f}{d x}$. Hence, if $m(x)=J^{2}(x)$, we have

$$
H=-\frac{1}{J^{2}} \frac{d^{2}}{d x^{2}}+\frac{2}{J^{2}}\left(\frac{d}{d x} \ln J\right) \frac{d}{d x}-\frac{2 a}{J^{2}}\left(\frac{d^{2}}{d x^{2}} \ln J\right)+4 a \frac{a+1}{J^{2}}\left(\frac{d}{d x} \ln J\right)^{2}+V,
$$

and

$$
A=-\frac{1}{J} \frac{d}{d x}+\frac{2 a+1}{J}\left(\frac{d}{d x} \ln J\right)+W
$$




$$
B=\frac{1}{J} \frac{d}{d x}+\frac{2 a}{J}\left(\frac{d}{d x} \ln J\right)+W
$$

In a similar way as in the constant mass case, it is not difficult to show that the expression (2) implies that the function $W$ must satisfy the Riccati equation ${ }^{28}$

$$
-\frac{1}{J} \frac{d W}{d x}+\frac{4 a+1}{J}\left(\frac{d}{d x} \ln J\right) W+W^{2}+\epsilon=V .
$$

Additionally, one may check that the operators $A, B$ fulfill the commutation rule

$$
[A, B]=-\frac{2}{J} \frac{d W}{d x}-\frac{4 a+1}{J^{2}}\left(\frac{d^{2}}{d x^{2}} \ln J\right)+\frac{4 a+1}{J^{2}}\left(\frac{d}{d x} \ln J\right)^{2} .
$$

Observe that the explicit form of $W$ can be determined from (8) if we impose a particular commutation rule for the factorizing operators. This will define the spectrum of the Hamiltonian, and the corresponding potential can be deduced from the Riccati equation (7). For instance, assuming that the operators $A, B$ fulfill the oscillator algebra $[B, A]=2$, we are immediately led to ${ }^{28}$

$$
W(x)=\int_{x_{0}}^{x} J(y) d y-\frac{4 a+1}{2 J}\left(\frac{d}{d x} \ln J\right),
$$

and, by setting $\epsilon=1$, we obtain a PDM oscillator potential

$$
V(x)=\left(\int_{x_{0}}^{x} J(y) d y\right)^{2}+\frac{4 a+1}{2 J^{2}(x)}\left(\frac{d^{2}}{d x^{2}} \ln J(x)\right)-(4 a+1) \frac{(4 a+3)}{4 J^{2}(x)}\left(\frac{d}{d x} \ln J(x)\right)^{2},
$$

for which the corresponding Hamiltonian will be isospectral to the harmonic oscillator.

In the next sections, we will follow this approach in order to explore the possibility of constructing different families of generalized PDM potentials of the Scarf type.

\section{3 | POSITION DEPENDENT MASS SCARF POTENTIALS}

Let us consider a hierarchy of PDM Hamiltonians $H_{\ell}$, labeled by a parameter $\ell=\ell_{\min }+s, s \in \mathbb{N}$, with $\ell_{\text {min }}>0$ a fixed real number

$$
H_{\ell}=-\frac{1}{J^{2}} \frac{d^{2}}{d x^{2}}+\frac{2}{J^{2}}\left(\frac{d}{d x} \ln J\right) \frac{d}{d x}-\frac{2 a}{J^{2}}\left(\frac{d^{2}}{d x^{2}} \ln J\right)+\frac{4 a}{J^{2}}(a+1)\left(\frac{d}{d x} \ln J\right)^{2}+V_{\ell}
$$

Let us also assume that the Hamiltonian (9) can be factorized in terms of 2 first order differential operators $A_{\ell}$, $B_{\ell}$ having the form

$$
\begin{gathered}
A_{\ell}=-\frac{1}{J} \frac{d}{d x}+\frac{2 a+1}{J}\left(\frac{d}{d x} \ln J\right)+W_{\ell} \\
B_{\ell}=\frac{1}{J} \frac{d}{d x}+\frac{2 a}{J}\left(\frac{d}{d x} \ln J\right)+W_{\ell}
\end{gathered}
$$

and fulfilling

$$
H_{\ell}=A_{\ell} B_{\ell}+\epsilon_{\ell}=B_{\ell-1} A_{\ell-1}+\epsilon_{\ell-1} .
$$

We will restrict ourselves to the description of bound states represented by normalized eigenfunctions $\psi_{\ell}^{n}$ of $H_{\ell}$. The corresponding eigenvalue equation reads

$$
H_{\ell} \psi_{\ell}^{n}=E_{\ell}^{n} \psi_{\ell}^{n}, \quad n=0,1,2, \cdots
$$

The condition (12) translates into the following set to 2 Riccati equations that must be satisfied simultaneously

$$
-\frac{1}{J} \frac{d W_{\ell}}{d x}+\frac{4 a+1}{J}\left(\frac{d}{d x} \ln J\right) W_{\ell}+W_{\ell}^{2}+\epsilon_{\ell}=V_{\ell}
$$




$$
\begin{aligned}
\frac{1}{J} \frac{d W_{\ell-1}}{d x} & +\frac{4 a+1}{J}\left(\frac{d}{d x} \ln J\right) W_{\ell-1}+W_{\ell-1}^{2} \\
& +\frac{4 a+1}{J^{2}}\left(\frac{d^{2}}{d x^{2}} \ln J\right)-\frac{4 a+1}{J^{2}}\left(\frac{d}{d x} \ln J\right)^{2}+\epsilon_{\ell-1}=V_{\ell} .
\end{aligned}
$$

To solve this system, we propose that

$$
W_{\ell}(x)=\phi(x)+\ell \beta(x),
$$

where $\phi$ and $\beta$ are 2 real-valued functions that do not dependent on the hierarchy label $\ell$. In this way, the set (14) and (15) transforms into the set

$$
\begin{gathered}
\frac{1}{J} \frac{d}{d x}\left[2 \phi+\frac{4 a+1}{J}\left(\frac{d}{d x} \ln J\right)\right]-\left[2 \phi+\frac{4 a+1}{J}\left(\frac{d}{d x} \ln J\right)\right] \beta=0 \\
\frac{1}{J} \frac{d \beta}{d x}-\beta^{2}=\frac{\epsilon_{\ell}-\epsilon_{\ell-1}}{2 \ell-1} .
\end{gathered}
$$

Observe that the right-hand side of (18) do not depend on the parameter $\ell$. This means that the corresponding left-hand side must be an $\ell$-independent constant $\alpha$ (compare with Kuru and Negro ${ }^{4}$ ).

\section{1 | Position dependent mass Scarf I-type potentials}

First, consider the case in which $\alpha>0$, ie, $\alpha=\kappa^{2}$ with $\kappa \in \mathbb{R}$. The expression

$$
\frac{\epsilon_{\ell}-\epsilon_{\ell-1}}{2 \ell-1}=\kappa^{2}
$$

implies that

$$
\epsilon_{\ell}=\kappa^{2} \ell^{2},
$$

and (18) is transformed into the Riccati equation

$$
\frac{1}{J} \frac{d \beta}{d x}-\beta^{2}=\kappa^{2}
$$

The general solution to this equation may be written as follows:

$$
\beta(x)=\kappa \tan \left(\kappa \int_{x_{0}}^{x} J(y) d y\right)-\frac{1}{J(x)} \frac{d}{d x} \ln \left[\Lambda-\frac{1}{\kappa} \tan \left(\kappa \int_{x_{0}}^{x} J(y) d y\right)\right],
$$

where $\Lambda$ is an arbitrary constant and $x_{0}$ is a constant with units of position that will be fixed in such a way that the function

$$
u(x)=\int_{x_{0}}^{x} J(y) d y
$$

fulfills the initial condition $u(0)=0$. As the function $u(x)$ may eventually take arbitrarily large values, the domain of validity of this solution will be defined by the value of $\Lambda$ in order to obtain nonsingular potentials. In this work, we consider the simplest case $\Lambda \rightarrow \infty$ leading to the particular solution

$$
\beta(x)=\kappa \tan (\kappa u(x)),
$$

which is valid in a domain given by $-\frac{\pi}{2}<\kappa u(x)<\frac{\pi}{2}$.

Next, by inserting the expression for $\beta$ into (17), we get

$$
\phi(x)=\kappa \lambda \sec (\kappa u(x))-\frac{4 a+1}{2 J(x)}\left(\frac{d}{d x} \ln J(x)\right),
$$

with $\lambda$ an arbitrary constant, and then, the expression for $W_{\ell}$ can be readily written

$$
W_{\ell}(x)=k \ell \tan (\kappa u(x))+\kappa \lambda \sec (\kappa u(x))-\frac{4 a+1}{2 J(x)}\left(\frac{d}{d x} \ln J(x)\right),
$$

along with those for the operators $A_{\ell}, B_{\ell}$

$$
A_{\ell}=-\frac{1}{J} \frac{d}{d x}+\frac{1}{2 J}\left(\frac{d}{d x} \ln J\right)+\kappa \lambda \sec (\kappa u)+\kappa \ell \tan (\kappa u),
$$




$$
B_{\ell}=\frac{1}{J} \frac{d}{d x}-\frac{1}{2 J}\left(\frac{d}{d x} \ln J\right)+\kappa \lambda \sec (\kappa u)+\kappa \ell \tan (\kappa u)
$$

Finally, we may consider either the Riccati equation (14) or (15) in order to obtain the potential $V_{\ell}$. After some calculations, we obtain a family of PDM trigonometric Scarf potentials given by

$$
\begin{aligned}
V_{\ell}(x)= & \kappa^{2}\left[\ell(\ell-1)+\lambda^{2}\right] \sec ^{2}(\kappa u(x))+(2 \ell-1) \kappa^{2} \lambda \sec (\kappa u(x)) \tan (\kappa u(x))+ \\
& +\frac{4 a+1}{2 J^{2}(x)}\left(\frac{d^{2}}{d x^{2}} \ln J(x)\right)-(4 a+1) \frac{(4 a+3)}{4 J^{2}(x)}\left(\frac{d}{d x} \ln J(x)\right)^{2} .
\end{aligned}
$$

This will be a confining potential for values of $\lambda$ in the interval $(-\ell+1, \ell-1)$. It is important to stress that the ordering parameter $a$ in this expression labels different forms of the potential, each one corresponding to a particular choice of the ordering of $m(x)$ and $P$ in the kinetic term. However, observe that the expressions (26) and (27) for the factorizing operators do not depend on this parameter. Then, the Hamiltonian $H_{\ell}$ will have the same form regardless the choice of the ordering in $T_{a}$.

The next step is the construction of the ground state $\psi_{\ell}^{0}(x)$ of the $\ell$-hierarchy. This is determined as the state that is annihilated by either $B_{\ell}$ or $A_{\ell-1}$ and that fulfills the square integrability condition. In this case, the solution with the correct behavior is annihilated by $B_{\ell}$. This means that this operator acts as an annihilator among the eigenfunctions of consecutive Hamiltonians. Hence, we set

$$
B_{\ell} \psi_{\ell}^{0}=\left[\frac{1}{J} \frac{d}{d x}-\frac{1}{2 J}\left(\frac{d}{d x} \ln J\right)+\kappa \lambda \sec (\kappa u)+\kappa \ell \tan (\kappa u)\right] \psi_{\ell}^{0}=0,
$$

to obtain

$$
\psi_{\ell}^{0}(x)=N_{\ell}^{0} \sqrt{J(x)}\left[\frac{\cos \left(\frac{\kappa u(x)}{2}\right)-\sin \left(\frac{\kappa u(x)}{2}\right)}{\cos \left(\frac{\kappa u(x)}{2}\right)+\sin \left(\frac{\kappa u(x)}{2}\right)}\right]^{\lambda} \cos ^{\ell}(\kappa u(x)),
$$

with

$$
N_{\ell}^{0}=2^{-\ell} \sqrt{\frac{\kappa \Gamma(2 \ell+1)}{\Gamma\left(\ell+\frac{1}{2}+\lambda\right) \Gamma\left(\ell+\frac{1}{2}-\lambda\right)}} .
$$

Also, one can see immediatelly from (12) and (13) that this state corresponds to an energy $E_{\ell}^{0}=\epsilon_{\ell}=\kappa^{2} \ell^{2}$.

On the other hand, the 2 factorizations (12) of $H_{\ell}$ lead to the following intertwining relations:

$$
A_{\ell-1} H_{\ell}=H_{\ell-1} A_{\ell-1}, \quad B_{\ell} H_{\ell}=H_{\ell+1} B_{\ell},
$$

so that it is possible to express the eigenfunctions of the Hamiltonians $H_{\ell \pm 1}$ in terms of those of $H_{\ell}$ by the proper application of $A_{\ell-1}$ and $B_{\ell}$. Indeed, if we consider the eigenvalue Equation 13, it is not difficult to show that the vector $A_{\ell-1} \psi_{\ell}^{n}$ is an eigenfunction of the Hamiltonian $H_{\ell-1}$ with eigenvalue $E_{\ell}^{n}$. Correspondingly, the function $B_{\ell} \psi_{\ell}^{n}$ is an eigenfunction of the Hamiltonian $\mathrm{H}_{\ell+1}$ with the same eigenvalue. These facts can be summarized by

$$
A_{\ell-1} \psi_{\ell}^{n} \propto \psi_{\ell-1}^{n+1}, \quad B_{\ell} \psi_{\ell}^{n} \propto \psi_{\ell+1}^{n-1},
$$

and $E_{\ell-1}^{n+1}=E_{\ell}^{n}=E_{\ell+1}^{n-1}$. In this way, it is possible to write

$$
E_{\ell}^{n}=E_{\ell+1}^{n-1}=\cdots=E_{\ell+n}^{0}=\kappa^{2}(\ell+n)^{2} .
$$

The proportionality constants for (32) can be determined by imposing the normalization condition on the wave functions $\psi_{\ell}^{n}$ and using the fact that $A_{\ell}$ and $B_{\ell}$ are adjoint to each other. We have

$$
A_{\ell-1} \psi_{\ell}^{n}=\kappa \sqrt{(n+1)(2 \ell+n-1)} \psi_{\ell-1}^{n+1}, \quad B_{\ell} \psi_{\ell}^{n}=\kappa \sqrt{(n)(2 \ell+n)} \psi_{\ell+1}^{n-1} .
$$

Moreover, the eigenstate $\psi_{\ell}^{n}(x)$ can be constructed from the ground state of the Hamiltonian $H_{\ell+n}$ by the subsequent application of the operators $A_{\ell}$ :

$$
\psi_{\ell}^{n}(x)=\frac{1}{\kappa^{n}} \sqrt{\frac{\Gamma(2 \ell+n)}{n ! \Gamma(2(\ell+n))}} A_{\ell} A_{\ell+1} \cdots A_{\ell+n-1} \psi_{\ell+n}^{0} .
$$




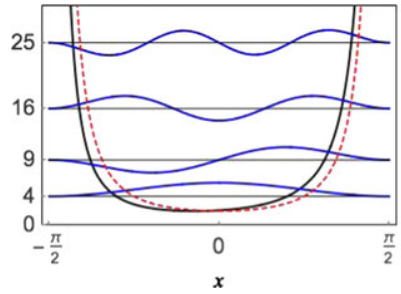

(A) $\ell=2, \lambda=0.3$

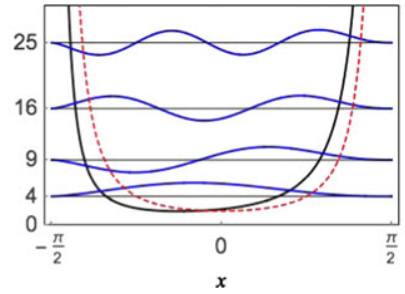

(B) $\ell=2, \lambda=0.6$

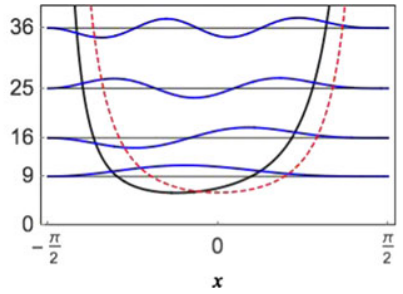

(C) $\ell=3, \lambda=0.9$

FIGURE 1 Trigonometric Scarf potentials $V_{\ell}$ and corresponding eigenfunctions for a constant mass $m=1$ and for the values of $\ell$ and $\lambda$ indicated. In all cases, $\kappa=1$. The red, dashed curve represents the corresponding symmetric Pöschl-Teller potential [Colour figure can be viewed at wileyonlinelibrary.com]

In this way, we finally arrive to the explicit expression for the eigenfunctions of a generic member $H_{\ell}$ of the hierarchy

$$
\psi_{\ell}^{n}(x)=N_{\ell}^{n} \sqrt{J(x)}\left[\frac{\cos \left(\frac{\kappa u(x)}{2}\right)-\sin \left(\frac{\kappa u(x)}{2}\right)}{\cos \left(\frac{\kappa u(x)}{2}\right)+\sin \left(\frac{\kappa u(x)}{2}\right)}\right]^{\lambda} \cos ^{\ell}(\kappa u(x)) P_{n}^{\left(\ell-\frac{1}{2}+\lambda, \ell-\frac{1}{2}-\lambda\right)}(\sin (\kappa u(x))),
$$

where the functions $P_{n}^{(\alpha, \beta)}(z)$ stand for the Jacobi polynomials of degree $n,{ }^{37}$ and the normalization constant is given by

$$
N_{\ell}^{n}=2^{-\ell} \kappa^{n} \sqrt{\frac{2 \kappa n !(\ell+n) \Gamma(2 \ell+n)}{\Gamma\left(\ell+n+\frac{1}{2}+\lambda\right) \Gamma\left(\ell+n+\frac{1}{2}-\lambda\right)}} .
$$

So far, we have constructed a family of PDM Scarf I potentials (28) labeled by the parameters $\ell$ and $\lambda$. In the case that $\lambda=0$, we recover the PDM Pöschl-Teller potentials already reported in Santiago-Cruz. ${ }^{29}$ In that case, the eigenfunctions take the form

$$
\psi_{\ell}^{n}(x)=\kappa^{n} \sqrt{\frac{2^{2 \ell-1}(\ell+n) n !}{\pi \Gamma(2(\ell+1))}} \Gamma(\ell) \sqrt{J(x)} \cos ^{\ell}(\kappa u(x)) C_{n}^{\ell}(\sin (\kappa u(x))) .
$$

with $C_{n}^{(\alpha)}(z)$ the Gegenbauer polynomials of degree $n$ and order $\alpha$, where we have used identity ${ }^{37}$

$$
P_{n}^{\left(\ell-\frac{1}{2}, \ell-\frac{1}{2}\right)}(z)=\frac{\Gamma(2 \ell) \Gamma\left(\ell+n+\frac{1}{2}\right)}{\Gamma(2 \ell+n) \Gamma\left(\ell+\frac{1}{2}\right)} C_{n}^{(\ell)}(z) .
$$

In the limit of constant mass, as $m \rightarrow 1$ and $u(x) \rightarrow x$, we obtain the family of Scarf I potentials (compare with De et al ${ }^{8}$ and Cooper et $\mathrm{al}^{9}$ )

$$
V_{\ell}(x)=\kappa^{2}\left[\ell(\ell-1)+\lambda^{2}\right] \sec ^{2}(\kappa x)+(2 \ell-1) \kappa \lambda \sec (\kappa x) \tan (\kappa x),
$$

from which the well-known trigonometric Pöschl-Teller family $V_{\ell}(x)=\kappa^{2} \ell(\ell-1) \sec ^{2}(\kappa x)$ can be recovered as a particular case when $\lambda=0 .{ }^{4,38}$

In Figure 1, we present some plots of the Scarf potentials (28) with few of their corresponding eigenfunctions, for the constant mass case $m=1$, and different values of $\ell$ and $\lambda$. In all the cases, the red, dashed curve corresponds to the symmetric Pöschl-Teller potential. In these plots, it is possible to observe how the potential deviates from the symmetric one as $\lambda$ take larger values while the energy levels remain unchanged.

Now, in order to present some examples, we consider different forms of $m(x)$ that were chosen in such a way that the function $u(x)$ take values in the whole real line as $x$ varies in $\operatorname{Dom}(m){ }^{24,39}$ Note that in all cases, the parameter $q$ allows to reach the limit of constant mass $m=1$ as $q \rightarrow 0$.

(i) Mass without singularities

First, consider the mass

$$
m_{r}(x)=\frac{1}{1+(q x)^{2}}
$$




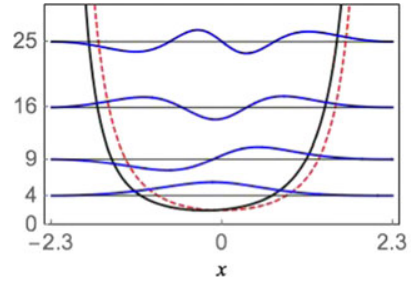

(A) $\ell=2, \lambda=0.3$

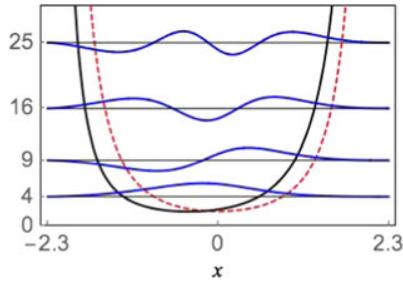

(B) $\ell=2, \lambda=0.5$

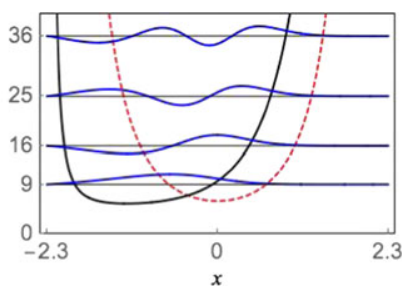

(C) $\ell=3, \lambda=1.9$

FIGURE 2 Position dependent mass Scarf I potentials $V_{\ell}$ for the mass $m_{r}(x)$ and some of its eigenfunctions for the values of $\ell$ and $\lambda$ indicated. In all cases, $\kappa=1$ and $q=1$. The red, dashed curve corresponds to the symmetric Pöschl-Teller case [Colour figure can be viewed at wileyonlinelibrary.com]

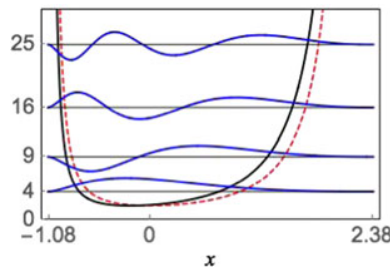

(A) $\ell=2, \lambda=0.3, q=\frac{1}{2}$

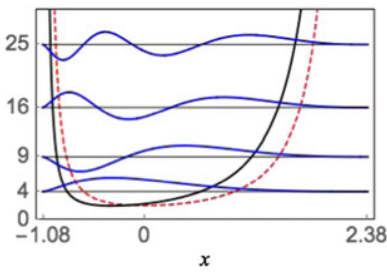

(B) $\ell=2, \lambda=0.5, q=\frac{1}{2}$

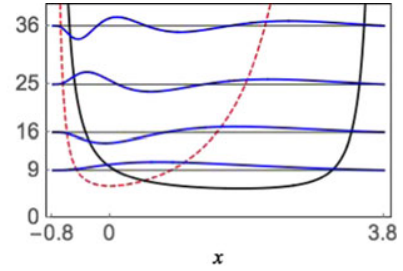

(C) $\ell=3, \lambda=-1.9, q=1$

FIGURE 3 Position dependent mass trigonometric Scarf potentials $V_{\ell}$ for the mass $m_{s}(x)$ and their first eigenfunctions, for the values of $\ell$, $\lambda$ and $q$ indicated. In all cases, $\kappa=1$. The red, dashed curve corresponds to the case $\lambda=0$ [Colour figure can be viewed at wileyonlinelibrary.com]

This is a bound function with domain of definition $\operatorname{Dom}\left(m_{r}\right)=\mathbb{R}$ having its maximum at $x=0$ and vanishing as $|x| \rightarrow \infty$. In this case, we are led to

$$
u_{r}(x)=\frac{1}{q} \operatorname{arcsinh}(q x)
$$

that ranges over all $\mathbb{R}$ for $x \in \mathbb{R}$.

In Figure 2, some graphics are presented to illustrate the PDM Scarf I potentials (28) when $m(x)=m_{r}(x)$. In all cases, the red, dashed curve represents the potential for the case $\lambda=0$. Observe that, as this parameter approaches its limits of validity, the potential barrier becomes sharper.

(ii) Mass with one singularity

Next, consider the mass

$$
m_{s}(x)=\frac{1}{(1+q x)^{2}} .
$$

For this mass, the domain $\operatorname{Dom}\left(m_{s}\right)=\left(-\frac{1}{q}, \infty\right)$. It becomes unbounded as $x \rightarrow-\frac{1}{q}$, and vanishes as $x \rightarrow \infty$. The corresponding $u$-function has now the form

$$
u_{s}(x)=\frac{1}{q} \ln (1+q x)
$$

ranging also over all $\mathbb{R}$.

The presence of a singularity in the mass may translate into a barrier in the corresponding potential if the singularity lies in the interval $-\frac{\pi}{2}<\kappa u(x)<\frac{\pi}{2}$. This fact should be taken into account in defining the domain of the PDM potential $\operatorname{Dom}\left(V_{\ell}\right)$. Figure 3 shows some plots for the potential (28) for the mass $m_{s}$. The red, dashed curve represents the PDM potential for $\lambda=0$. Observe the potential barrier at the position of the singularity that becomes sharper as the value of $q$ grows. The black, continuous curves represent the potential $V_{\ell}$ for different values of $\ell$ and $\lambda$. Note the deformation of the potential and the corresponding eigenfunctions due to variation of the parameter $\lambda$.

(iii) Mass with 2 singularities

Finally, consider the mass

$$
m_{s s}(x)=\frac{1}{\left(1-(q x)^{2}\right)^{2}},
$$




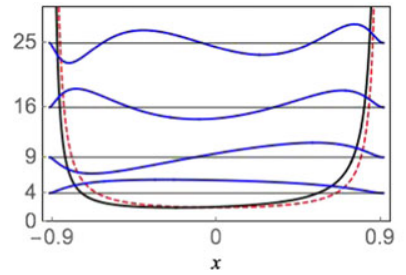

(A) $\ell=2, \lambda=0.3, q=1$

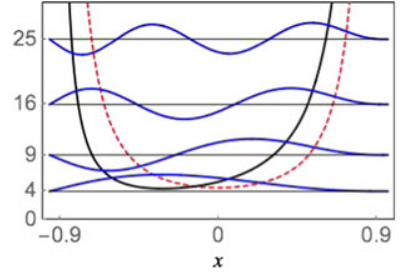

(B) $\ell=3, \lambda=1.9, q=1$

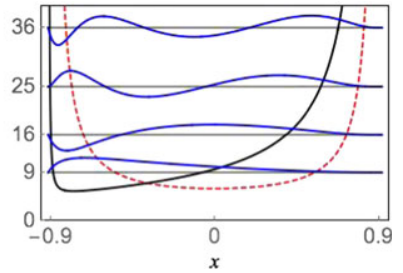

(C) $\ell=2, \lambda=0.9, q=\frac{1}{2}$

FIGURE 4 Position dependent mass trigonometric Scarf potentials $V_{\ell}$ for the mass $m_{s s}(x)$ and their first eigenfunctions for the values of $\ell$, $\lambda$, and $q$ indicated. The plots (A) and (B) correspond to $\kappa=1$ while (C) to $\kappa=1.5$. The red curve corresponds to the symmetric case $\lambda=0$ [Colour figure can be viewed at wileyonlinelibrary.com]

with a bound domain $\operatorname{Dom}\left(m_{s s}\right)=\left(-\frac{1}{q}, \frac{1}{q}\right)$. In this case,

$$
u_{s s}(x)=\frac{1}{q} \arctan (q x)
$$

The corresponding graphics of $V_{\ell}$ and their corresponding wave functions can be appreciated in Figure 4 for different values of $\ell, \lambda$, and $q$.

\subsection{Trigonometric Scarf potential algebra and hierarchies}

To close this section, we comment something about the underlying potential algebra for these systems. First, let us introduce the free index operators $A, B, C$ such that

$$
\begin{gathered}
A \psi_{\ell}^{n}=A_{\ell-1} \psi_{\ell}^{n}=\kappa \sqrt{(n+1)(2 \ell+n-1)} \psi_{\ell-1}^{n+1}, \\
B \psi_{\ell}^{n}=B_{\ell} \psi_{\ell}^{n}=\kappa \sqrt{n(2 \ell-n)} \psi_{\ell+1}^{n-1} \\
C \psi_{\ell}^{n}=\frac{1}{2}\left(A_{\ell} B_{\ell}-B_{\ell-1} A_{\ell-1}\right) \psi_{\ell}^{n}=\kappa^{2}\left(\frac{1}{2}-\ell\right) \psi_{\ell}^{n} .
\end{gathered}
$$

It is not difficult to show that these operators fulfill the commutation relations

$$
[B, A]=-2 C, \quad[C, A]=\kappa^{2} A, \quad[C, B]=-\kappa^{2} B .
$$

This means that we may construct a realization of the generators of the $s u(2)$ algebra with the following identification:

$$
\mathcal{J}_{0}=\frac{1}{\kappa^{2}} C, \quad \mathcal{J}_{-}=\frac{1}{\kappa} B, \quad \mathcal{J}_{+}=\frac{1}{\kappa} A,
$$

as these operators fulfill the $s u(2)$ algebra (compare with Kuru and Negro ${ }^{4}$ )

$$
\left[\mathcal{J}_{-}, \mathcal{J}_{+}\right]=-2 \mathcal{J}_{0}, \quad\left[\mathcal{J}_{0}, \mathcal{J}_{ \pm}\right]= \pm \mathcal{J}_{ \pm}
$$

In the $(\ell, n)$-plane of parameters defining the eigenstates of the $\ell$-hierarchies, the action of the operators $\mathcal{J}_{ \pm}$induce the mappings $(\ell, n) \rightarrow(\ell \mp 1, n \pm 1)$ meaning that the states are transformed in such a way that the quantity $\ell+n$ is preserved. This unveil the fact that the full set of states may be also classify into hierarchies each one composed of all states having the same value of this quantity. Introducing the parameter $j=\frac{1}{2}\left(\ell-\ell_{\min }+n-1\right)$, we say that the operators $\mathcal{J}_{ \pm}$intertwine eigenstates corresponding to the same $j$-hierarchy. For each fixed value of $\ell_{\min }$, the complete space of wave functions $\psi_{\ell}^{n}$ splits into the direct sum of the set of subspaces $\left\{\mathcal{H}^{j}, j=0, \frac{1}{2}, 1, \cdots\right\}$ spanned by the corresponding hierarchies. This fact is relevant, eg, in the construction of different families of coherent states and in the generation of the dynamical algebra underlying the corresponding system..$^{4,40-42}$

\subsection{Position dependent mass Scarf II-type potentials}

Now, suppose that $\alpha<0$, ie, $\alpha=-\kappa^{2}$. Thus,

$$
\epsilon_{\ell}=-\kappa^{2} \ell^{2},
$$


and the Riccati equation (18) turns into

$$
\frac{1}{J} \frac{d \beta}{d x}-\beta^{2}=-\kappa^{2}
$$

The general solution of this equation has the form

$$
\beta(x)=-\kappa \tanh (\kappa u(x))-\frac{1}{J(x)} \frac{d}{d x} \ln \left[\Lambda-\frac{1}{\kappa} \tanh (\kappa u(x))\right],
$$

which is valid for values of $\Lambda$ such that $|\Lambda| \geq \frac{1}{\kappa}$. As in the trigonometric case, we will focus on the simplest particular solution for which $|\Lambda| \rightarrow \infty$, ie,

$$
\beta(x)=-\kappa \tanh (\kappa u(x)) .
$$

As this is a regular function for $x \in \mathbb{R}$, the domain of definition in this case will be only determined by the corresponding $\operatorname{Dom}(m)$. The $\phi$-function now take the form

$$
\phi(x)=\kappa \lambda \operatorname{sech}(\kappa u(x))-\frac{4 a+1}{2 J(x)}\left(\frac{d}{d x} \ln J(x)\right),
$$

and leads to the function $W_{\ell}$

$$
W_{\ell}(x)=-\kappa \ell \tanh (\kappa u(x))+\kappa \lambda \operatorname{sech}(\kappa u(x))-\frac{4 a+1}{2 J(x)}\left(\frac{d}{d x} \ln J(x)\right),
$$

along with the operators $A_{\ell}, B_{\ell}$

$$
\begin{gathered}
A_{\ell}=-\frac{1}{J} \frac{d}{d x}+\frac{1}{2 J}\left(\frac{d}{d x} \ln J\right)+\kappa \lambda \operatorname{sech}(\kappa u)-\kappa \ell \tanh (\kappa u), \\
B_{\ell}=\frac{1}{J} \frac{d}{d x}-\frac{1}{2 J}\left(\frac{d}{d x} \ln J\right)+\kappa \lambda \operatorname{sech}(\kappa u)-\kappa \ell \tanh (\kappa u),
\end{gathered}
$$

which are, consistently, independent of the ordering parameter $a$. As in the trigonometric case, the Ricatti equation (14) allows us to obtain the family of PDM Scarf II (hyperbolic) potentials. We get

$$
\begin{aligned}
V_{\ell}(x)= & \kappa^{2}\left[-\ell(\ell-1)+\lambda^{2}\right] \operatorname{sech}^{2}(\kappa u(x))-(2 \ell-1) \kappa \lambda \operatorname{sech}(\kappa u(x)) \tanh (\kappa u(x))+ \\
& +\frac{4 a+1}{2 J^{2}(x)}\left(\frac{d^{2}}{d x^{2}} \ln J(x)\right)-(4 a+1) \frac{(4 a+3)}{4 J^{2}(x)}\left(\frac{d}{d x} \ln J(x)\right)^{2} .
\end{aligned}
$$

In the constant mass case limit, we obtain the family of Scarf II potentials

$$
V_{\ell}(x)=\kappa^{2}\left[-\ell(\ell-1)+\lambda^{2}\right] \operatorname{sech}^{2}(\kappa x)-(2 \ell-1) \kappa \lambda \operatorname{sech}(\kappa x) \tanh (\kappa x) .
$$

These potentials have been considered, eg, in constructing exact solutions of the Schrödinger or Dirac equations by using either power series, ${ }^{43}$ or shape-invariance ${ }^{8,9,44}$ techniques. In the context of non-Hermitian Hamiltonians, the complexification of the Scarf II-type potential has been also considered from the group theoretical point of view. ${ }^{45-47}$ Figure 5 shows some plots of potential (59) together with their first eigenfunctions for different values of the parameters.

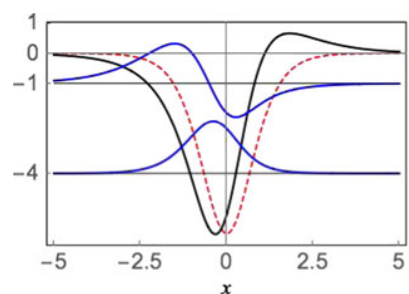

$$
\text { (A) } \ell=3, \lambda=-0.8
$$

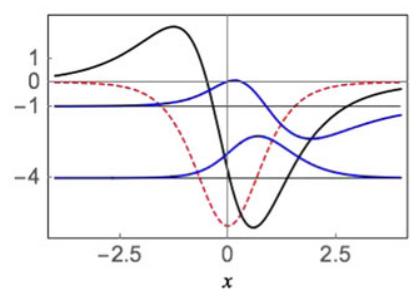

(B) $\ell=3, \lambda=1.5$

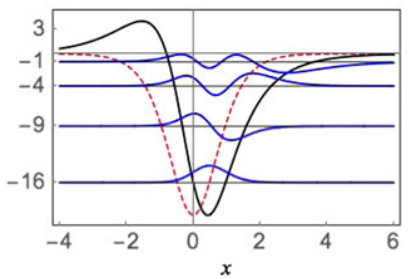

(C) $\ell=3, \lambda=2$

FIGURE 5 Hyperbolic Scarf potentials for a constant mass $m=1$ given by (43) for the values of $\ell$ and $\lambda$ indicated. In all cases, $\kappa=1$. The red, dashed curve corresponds to the symmetric constant mass hyperbolic Pöschl-Teller potential [Colour figure can be viewed at wileyonlinelibrary.com] 
Unlike the trigonometric case, for the Scarf II potential, the ground state $\psi_{\ell}^{0}$ of the $\ell$-hierarchy is constructed as that state that is annihilated by the operator $A_{\ell-1}$, rather than $B_{\ell}$. This means that the operators $A_{\ell}$ will act as annihilators among the elements of consecutive Hamiltonians. Hence,

$$
A_{\ell-1} \psi_{\ell}^{0}=\left[-\frac{1}{J} \frac{d}{d x}+\frac{1}{2 J}\left(\frac{d}{d x} \ln J\right)+\kappa \lambda \operatorname{sech}(\kappa u)-\kappa(\ell-1) \tanh (\kappa u)\right] \psi_{\ell}^{0}=0
$$

After some calculations, we get

$$
\psi_{\ell}^{0}(x)=N_{\ell}^{0} \sqrt{J(x)}[\cosh (\kappa u(x))]^{-\ell+1} \exp \{\lambda \arctan [\sinh (\kappa u(x))]\},
$$

with the normalization constant

$$
N_{\ell}^{0}=\sqrt{\frac{\kappa \Gamma\left(\ell-\frac{1}{2}+i \lambda\right) \Gamma\left(\ell-\frac{1}{2}-i \lambda\right)}{\sqrt{\pi} \Gamma(\ell-1) \Gamma\left(\ell-\frac{1}{2}\right)}} .
$$

As this state is annihilated by $A_{\ell-1}$, it corresponds to the eigenvalue is $E_{\ell}^{n}=\epsilon_{\ell-1}=-\kappa^{2}(\ell-1)^{2}$.

The intertwining relations (31) determine the action of the $A_{\ell}$ and $B_{\ell}$ operators on the wave functions among different Hamiltonians, indeed

$$
A_{\ell-1} \psi_{\ell}^{n} \propto \psi_{\ell-1}^{n-1}, \quad B_{\ell} \psi_{\ell}^{n} \propto \psi_{\ell+1}^{n+1},
$$

with the condition that $E_{\ell-1}^{n-1}=E_{\ell}^{n}=E_{\ell+1}^{n+1}$. In this way, we may state that

$$
E_{\ell}^{n}=E_{\ell-1}^{n-1}=\cdots=E_{\ell-n}^{0}=-\kappa^{2}(\ell-n-1)^{2} .
$$

The value of $E_{\ell}^{0}$ allows now to determine the proportionality constants for the expressions (62), we have

$$
A_{\ell-1} \psi_{\ell}^{n}=\kappa \sqrt{n(2 \ell-n-2)} \psi_{\ell-1}^{n-1}, \quad B_{\ell} \psi_{\ell}^{n}=\kappa \sqrt{(n+1)(2 \ell-n-1)} \psi_{\ell+1}^{n+1} .
$$

Additionally, the wave function $\psi_{\ell}^{n}(x)$ can be determined from the ground state of the $(\ell-n)$-Hamiltonian by the proper subsequent applications of operators $B_{\ell}$. Indeed,

$$
\psi_{\ell}^{n}(x)=\frac{1}{\kappa^{n}} \sqrt{\frac{\Gamma(2 \ell-2 n-1)}{n ! \Gamma(2 \ell-n-1)}} B_{\ell-1} B_{\ell-2} \cdots B_{\ell-n} \psi_{\ell-n}^{0}(x) .
$$

Finally, the expression for the eigenfunctions of a generic member of the hierarchy come along

$$
\begin{aligned}
\psi_{\ell}^{n}(x)= & N_{\ell}^{n} \sqrt{J(x)}[\cosh (\kappa u(x))]^{-\ell+1} \exp \{\lambda \arctan [\sinh (\kappa u(x))]\} \times \\
& \times P_{n}^{\left(-\ell+\frac{1}{2}+i \lambda,-\ell+\frac{1}{2}-i \lambda\right)}(i \sinh (\kappa u(x))),
\end{aligned}
$$

with the normalization constant

$$
N_{\ell}^{n}=(-i)^{n} 2^{\ell-1} \sqrt{\frac{\kappa n !(\ell-n-1) \Gamma\left(\ell-n-\frac{1}{2}+i \lambda\right) \Gamma\left(\ell-n-\frac{1}{2}-i \lambda\right)}{\pi \Gamma(2 \ell-n-1)}} .
$$

Figures 6 to 8 show some plots of the PDM Scarf II potentials (58) and its first wave functions for the masses $m_{r}, m_{s}$, and $m_{s s}$, respectively, and for diverse values of the parameters. In all cases, the red, dashed curve represent the corresponding symmetric Pöschl-Teller case for $\lambda=0$. The description of the behavior of these potentials and the eigenfunctions is similar to that presented in Section 3.1.

\subsection{Hyperbolic Scarf potential algebra and hierarchies}

Let us say a few words about the underlying algebra of the Scarf II potential. As in the trigonometric case, it is possible to define the free index operators $A, B$ given by

$$
A \psi_{\ell}^{n}=A_{\ell-1} \psi_{\ell}^{n}=\kappa \sqrt{n(2 \ell-n-2)} \psi_{\ell-1}^{n-1}
$$




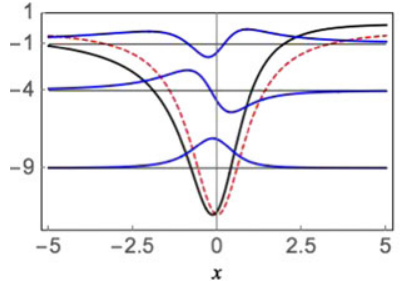

(A) $\ell=4, \lambda=-0.5$

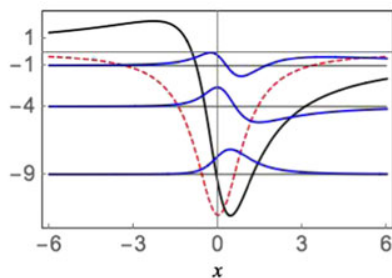

(B) $\ell=4, \lambda=1.5$

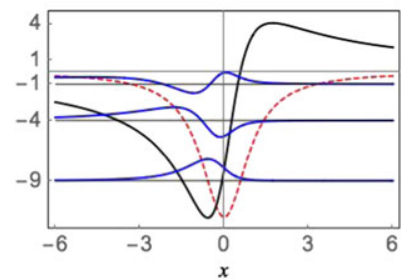

(C) $\ell=4, \lambda=-2$

FIGURE 6 Position dependent mass Scarf II potentials for the regular mass $m_{r}$ given by (39) with the values of $\ell$ and $\lambda$ indicated. In all cases, $q=1$ and $\kappa=1$. The red, dashed curve corresponds to the symmetric Pöschl-Teller case [Colour figure can be viewed at wileyonlinelibrary.com]

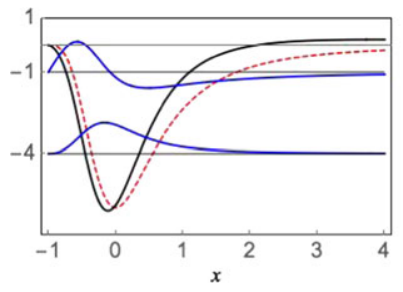

(A) $\ell=3, \lambda=-0.3, \kappa=1.5$

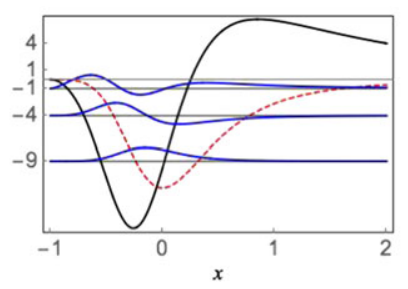

(B) $\ell=4, \lambda=-1.5, \kappa=2$

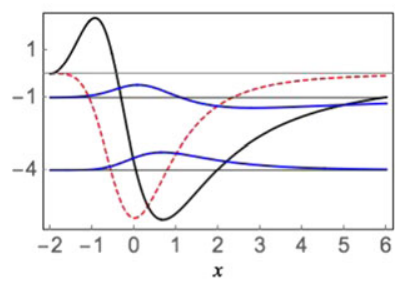

(C) $\ell=3, \lambda=1.5, \kappa=1$.

FIGURE 7 Position dependent mass Scarf II potentials for the singular mass $m_{s}$ given in (41) with the values of $\ell$, $\lambda$, and $\kappa$ indicated. The plots (A) and (B) correspond to $q=1$ while (C) to $\lambda=\frac{1}{2}$. In all cases, the red, dashed curve represents the case $\lambda=0$ [Colour figure can be viewed at wileyonlinelibrary.com]

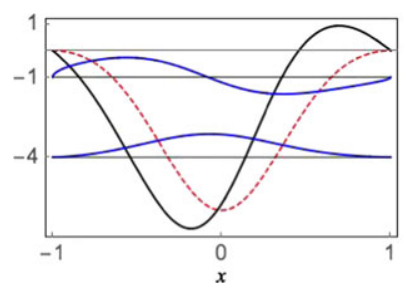

(A) $\ell=3, \lambda=-0.5, \kappa=2$

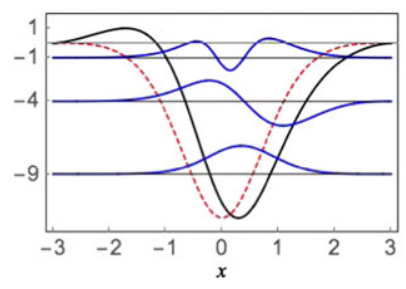

(B) $\ell=4, \lambda=1, \kappa=1$

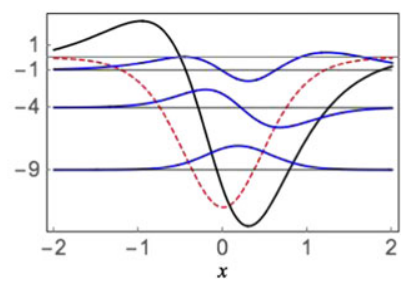

(C) $\ell=4, \lambda=1.2, \kappa=1.5$

FIGURE 8 Position dependent mass Scarf II potentials for a constant mass $m_{s S}$ given in (43) with the values of $\ell$, $\lambda$, and $\kappa$ indicated. The plot (A) correspond to $q=1$, while (B) and (C) to $q=\frac{1}{3}$. The red, dashed curve corresponds to $\lambda=0$ [Colour figure can be viewed at wileyonlinelibrary.com]

$$
\begin{gathered}
B \psi_{\ell}^{n}=B_{\ell} \psi_{\ell}^{n}=\kappa \sqrt{(n+1)(2 \ell-n-1)} \psi_{\ell+1}^{n+1} \\
C \psi_{\ell}^{n}=\frac{1}{2}\left(B_{\ell-1} A_{\ell-1}-A_{\ell} B_{\ell}\right) \psi_{\ell}^{n}=\kappa^{2}\left(\frac{1}{2}-\ell\right) \psi_{\ell}^{n} .
\end{gathered}
$$

One can easily show that these operators fulfill the commutation relations

$$
[B, A]=2 C, \quad[C, A]=\kappa^{2} A, \quad[C, B]=-\kappa^{2} B .
$$

Thus, the introduction of new operators $\mathcal{K}_{0}$ and $\mathcal{K}_{ \pm}$of the form

$$
\mathcal{K}_{0}=\frac{1}{\kappa^{2}} C, \quad \mathcal{K}_{-}=\frac{1}{\kappa} B, \quad \mathcal{K}_{+}=\frac{1}{\kappa} A,
$$

leads us to a realization of the $\mathrm{su}(1,1)$ algebra (compare with Kuru and $\mathrm{Negro}^{4}$ )

$$
\left[\mathcal{K}_{-}, \mathcal{K}_{+}\right]=2 \mathcal{K}_{0}, \quad\left[\mathcal{K}_{0}, \mathcal{K}_{ \pm}\right]= \pm \mathcal{K}_{ \pm}
$$

The action of the operators $\mathcal{K}_{0}, \mathcal{K}_{ \pm}$in the $(\ell, n)$-plane of parameters induce the mappings $(\ell, n) \rightarrow(\ell \pm 1, n \pm 1)$. Observe that in these transformation the quantity $\ell-n$ remains invariant. Let us introduce the parameter $k=\frac{1}{2}\left(\ell-\ell_{\min }-n+1\right)$. 
The whole set of wave functions $\psi_{\ell}^{n}$ may be classified into hierarchies composed by those vectors having the same value of $k$. The operators $\mathcal{K}_{ \pm}$intertwine the wave functions corresponding to the same $k$-hierarchy. Thus, for each fixed value of $\ell_{\text {min }}$, the whole set of wave functions $\psi_{\ell}^{n}$ can be decomposed as the direct sum of the subspaces $\left\{\mathcal{H}^{k}, k=1, \frac{3}{2}, 2 \cdots\right\}$ each one spanned by the corresponding $k$-hierarchy. ${ }^{41,42}$

\section{POSITION DEPENDENT MASS POTENTIALS WITH THE SCARF SPECTRUM GENERATED BY SUPERSYMMETRY}

\section{1 | Potentials with the trigonometric Scarf spectrum}

Now, a natural question arises, if there exist more general operators, say, $\widetilde{A}_{\ell}, \widetilde{B}_{\ell}$ that allow to factorize the PDM Hamiltonian $H_{\ell}$ in the form

$$
H_{\ell}=\widetilde{B}_{\ell-1} \widetilde{A}_{\ell-1}+\epsilon .
$$

The answer is positive. Indeed, the solution to the Schrödinger equation

$$
H_{\ell} \psi=\left\{m^{a} \mathrm{Pm}^{2 b} \mathrm{Pm} m^{a}+V_{\ell}\right\} \psi=\epsilon \psi,
$$

with $V_{\ell}$ a member of the PDM Scarf I family (28), can be written in the form (from now on, for the sake of simplicity, and without loss of generality, we will set $a=-\frac{1}{4}$ )

$$
\psi(x)=\sqrt{J(x)} \exp \left[\int^{x} \widetilde{W}_{\ell-1}(y) J(y) d y\right],
$$

where the function $\widetilde{W}_{\ell-1}(x)$ fulfills the Riccati equation

$$
\frac{1}{J} \frac{d}{d x} \widetilde{W}_{\ell-1}(x)+\widetilde{W}_{\ell-1}^{2}(x)+\epsilon=V_{\ell}(x),
$$

that has the same form as (15) for $a=-\frac{1}{4}$. This means that the Hamiltonian $H_{\ell}$ can be factorized in the form (72) with

$$
\begin{gathered}
\widetilde{A}_{\ell-1}=-\frac{1}{J} \frac{d}{d x}+\frac{1}{2 J}\left(\frac{d}{d x} \ln J\right)+\widetilde{W}_{\ell-1} \\
\widetilde{B}_{\ell-1}=\frac{1}{J} \frac{d}{d x}-\frac{1}{2 J}\left(\frac{d}{d x} \ln J\right)+\widetilde{W}_{\ell-1} .
\end{gathered}
$$

In general, for an arbitrary choice of one solution $\widetilde{W}_{\ell-1}$ of the Riccati equation (75), the corresponding operators $\widetilde{A}_{\ell-1}, \widetilde{B}_{\ell-1}$ do not fulfill the Scarf potential algebra (12). In fact, if we reverse the ordering of the factors in (72), we obtain a new Hamiltonian $\widetilde{H}_{\ell-1}$ given by

$$
\begin{aligned}
\widetilde{H}_{\ell-1} & =\widetilde{A}_{\ell-1} \widetilde{B}_{\ell-1}+\epsilon \\
& =-\frac{1}{J^{2}} \frac{d^{2}}{d x^{2}}+\frac{2}{J^{2}}\left(\frac{d}{d x} \ln J\right) \frac{d}{d x}+\frac{1}{2 J^{2}}\left(\frac{d^{2}}{d x^{2}} \ln J\right)-\frac{3}{4 J^{2}}\left(\frac{d}{d x} \ln J\right)^{2}+\widetilde{V}_{\ell-1},
\end{aligned}
$$

where the new potential $\widetilde{V}_{\ell-1}$ is defined by

$$
\widetilde{V}_{\ell-1}(x)=V_{\ell}(x)-\frac{2}{J}\left(\frac{d}{d x} \widetilde{W}_{\ell-1}(x)\right) .
$$

Now, the factorizations (72) and (78) imply the intertwining relations

$$
\widetilde{H}_{\ell-1} \widetilde{A}_{\ell-1}=\widetilde{A}_{\ell-1} H_{\ell}, \quad H_{\ell} \widetilde{B}_{\ell-1}=\widetilde{B}_{\ell-1} \widetilde{H}_{\ell-1},
$$

that, in turn allow to construct the wave functions of $\widetilde{H}_{\ell-1}$ in terms of those of $H_{\ell}$. Note that the first one of the intertwining relations (80) implies that the function $\widetilde{A}_{\ell-1} \psi_{\ell}^{n}$ is an eigenfunction of $\widetilde{H}_{\ell-1}$ corresponding to the eigenvalue $E_{\ell}^{n}$, while the second one establishes that $B_{\ell-1}$ reverses the action of $A_{\ell-1}$.

It may happen, however, as it is well known for the constant mass case, that the functions $\widetilde{A}_{\ell-1} \psi_{\ell}^{n}$ do not conform a complete set of eigenfunctions of $\widetilde{H}_{\ell-1}$. If this is the case, there is only one additional wave function $\widetilde{\psi}_{\ell-1}^{\epsilon}$ that is normalizable in $\operatorname{Dom}\left(\widetilde{V}_{\ell-1}\right)$. This “missing" state is determined as the function that is annihilated by $\widetilde{B}_{\ell-1}$ and, thus, corresponds 
to the eigenvalue $\epsilon$. In this way, if $\epsilon \neq E_{\ell}^{n}, n=0,1,2, \ldots$, the Hamiltonians $H_{\ell}$ and $\widetilde{H}_{\ell-1}$ are almost isospectral and $S p\left(\widetilde{H}_{\ell-1}\right)=S p\left(H_{\ell}\right) \cup\{\epsilon\}$. To the contrary, if the equation $\widetilde{B}_{\ell-1} \widetilde{\psi}_{\ell-1}^{\epsilon}=0$ do not define any square integrable function $\widetilde{\psi}_{\ell}^{\epsilon}$ in the corresponding domain, then the set $\left\{\widetilde{A}_{\ell-1} \psi_{\ell}^{n}, n=0,1,2, \cdots\right\}$ is a complete set of eigenfunctions of $\widetilde{H}_{\ell-1}$ and $\operatorname{Sp}\left(\widetilde{H}_{\ell-1}\right)=S p\left(H_{\ell}\right)$.

As an example, take the particular case for which $\epsilon=\epsilon_{\ell-1}=\kappa^{2}(\ell-1)^{2}$. Following (16) and (24), we propose that

$$
\widetilde{W}_{\ell-1}(x)=\kappa \lambda \sec (\kappa u(x))+(\ell-1) \widetilde{\beta}(x) .
$$

Then, the function $\widetilde{\beta}$ must satisfy the Riccati equation

$$
\frac{1}{J} \frac{d \widetilde{\beta}}{d x}+2 \kappa \lambda \widetilde{\beta}+(\ell-1) \widetilde{\beta}^{2}+(\ell-1) \kappa^{2}=\kappa^{2} \ell \sec ^{2}(\kappa u)+2 \kappa \lambda \sec (\kappa u) \tan (\kappa u) .
$$

The general solution to this equation reads

$$
\widetilde{\beta}(x)=\kappa \tan (\kappa u(x))+\frac{1}{(\ell-1) J(x)} \frac{d}{d x} \ln \left[\gamma+(\ell-1) \int^{x} \mu(y) J(y) d y\right],
$$

with $\gamma$ an integration constant and

$$
\mu(x)=(1-\sin (\kappa u(x)))^{\ell+\lambda-1}(1+\sin (\kappa u(x)))^{\ell-\lambda-1} .
$$

This expression, together with (79) allows to construct a family of supersymmetric potentials labeled by the parameter $\gamma$ :

$$
\begin{aligned}
\widetilde{V}_{\ell-1}(x)=V_{\ell-1}(x) & +\frac{2}{J^{2}(x)}\left(\frac{d}{d x} \ln J\right) \frac{d}{d x} \ln \left[\gamma+(\ell-1) \int^{x} \mu(y) J(y) d y\right]+ \\
& -\frac{2}{J^{2}(x)} \frac{d^{2}}{d x^{2}} \ln \left[\gamma+(\ell-1) \int^{x} \mu(y) J(y) d y\right] .
\end{aligned}
$$

To obtain regular potentials the parameter $\gamma$ should be restricted by the condition $|\gamma|>\max \left[(\ell-1) \int^{x} \mu(y) J(y) d y\right]$.

As the constant $\epsilon=\epsilon_{\ell-1}=\kappa^{2}(\ell-1)^{2}$ do not belong to $S p\left(H_{\ell}\right)$, we look for a missing state corresponding to this eigenvalue by imposing the condition that $B_{\ell-1} \widetilde{\psi}_{\ell-1}^{\epsilon}=0$. We find the solution

$$
\widetilde{\psi}_{\ell-1}^{\epsilon}(x) \propto \sqrt{J(x)}\left[\frac{\cos \left(\frac{\kappa u(x)}{2}\right)-\sin \left(\frac{\kappa u(x)}{2}\right)}{\cos \left(\frac{\kappa u(x)}{2}\right)+\sin \left(\frac{\kappa u(x)}{2}\right)}\right]^{\lambda} \frac{[\cos (\kappa u(x))]^{\ell-1}}{\gamma+(\ell-1) \int^{x} \mu(y) J(y) d y},
$$

which is a normalizable function in $\operatorname{Dom}\left(\widetilde{V}_{\ell-1}\right)$. This function must be then added to the set of eigenfunctions of $\widetilde{H}_{\ell-1}$ and the corresponding eigenvalue to its spectrum. Thus, $S p\left(\widetilde{H}_{\ell-1}\right)=S p\left(H_{\ell}\right) \cup\left\{\kappa^{2}(\ell-1)^{2}\right\}$, or $S p\left(\widetilde{H}_{\ell-1}\right)=S p\left(H_{\ell-1}\right)$.

\section{2 | Potentials with the hyperbolic Scarf spectrum}

Now, consider the PDM Schrödinger equation (73) with $V_{\ell}$ a member of the Scarf II family (58). It is possible to write the solution in the form

$$
\psi(x)=\sqrt{J(x)} \exp \left[-\int^{x} \widetilde{W}_{\ell}(y) J(y) d y\right],
$$

where the function $\widetilde{W}_{\ell}$ must now satisfy the Riccati equation

$$
-\frac{1}{J} \frac{d \widetilde{W}_{\ell}}{d x}+\widetilde{W}_{\ell}^{2}+\epsilon=V_{\ell}
$$

Note that this equation is the same as Equation 14 for $a=-\frac{1}{4}$. This means that we may factorize the Hamiltonian $H_{\ell}$ in the form

$$
H_{\ell}=\widetilde{A}_{\ell} \widetilde{B}_{\ell}+\epsilon,
$$

with

$$
\widetilde{A}_{\ell}=-\frac{1}{J} \frac{d}{d x}+\frac{1}{2 J}\left(\frac{d}{d x} \ln J\right)+\widetilde{W}_{\ell}
$$




$$
\widetilde{B}_{\ell}=\frac{1}{J} \frac{d}{d x}-\frac{1}{2 J}\left(\frac{d}{d x} \ln J\right)+\widetilde{W}_{\ell}
$$

In a similar way as in the previous case, in general, not any solution to (87) lead to the fulfillment of the Scarf potential algebra (12). In fact, if we reverse the product in (88), we obtain the new Hamiltonian

$$
\begin{aligned}
\widetilde{H}_{\ell+1} & =\widetilde{B}_{\ell} \widetilde{A}_{\ell}+\epsilon \\
& =-\frac{1}{J^{2}} \frac{d^{2}}{d x^{2}}+\frac{2}{J^{2}}\left(\frac{d}{d x} \ln J\right) \frac{d}{d x}+\frac{1}{2 J^{2}}\left(\frac{d^{2}}{d x^{2}} \ln J\right)-\frac{3}{4 J^{2}}\left(\frac{d}{d x} \ln J\right)^{2}+\widetilde{V}_{\ell+1},
\end{aligned}
$$

where the new potential

$$
\widetilde{V}_{\ell+1}(x)=\widetilde{V}_{\ell}(x)+\frac{1}{2 J(x)} \frac{d}{d x} \widetilde{W}_{\ell}(x)
$$

Now, the intertwining relations

$$
\widetilde{B}_{\ell} H_{\ell}=\widetilde{H}_{\ell+1} \widetilde{B}_{\ell}, \quad \widetilde{A}_{\ell} \widetilde{H}_{\ell+1}=H_{\ell} \widetilde{A}_{\ell},
$$

establish that one may construct the wave functions of $\widetilde{H}_{\ell+1}$ in terms of those of $H_{\ell}$. Actually, these relations imply that the vector $\widetilde{B}_{\ell} \psi_{\ell}^{n}$ is an eigenfunction of $\widetilde{H}_{\ell+1}$ corresponding to the eigenvalue $E_{\ell}^{n}$ and that $\widetilde{A}_{\ell}$ reverses the effect of $\widetilde{B}_{\ell}$.

Also, in the case that $\epsilon \neq E_{\ell}^{n}, n=0,1,2, \ldots$, if $\left\{\tilde{B}_{\ell} \psi_{\ell}^{n}, n=0,1,2, \cdots\right\}$ is not a complete set of eigenfunctions of $\widetilde{H}_{\ell+1}$, there is only one missing normalizable state $\widetilde{\psi}_{\ell+1}^{\epsilon}$, which is constructed by assuming that $\widetilde{A}_{\ell} \widetilde{\psi}_{\ell+1}^{\epsilon}=0$. As this state corresponds to the eigenvalue $\epsilon$, this value must be added to the spectrum of $\widetilde{H}_{\ell+1}$, and the Hamiltonians $H_{\ell}$ and $H_{\ell+1}$ are almost isospectral: $S p\left(\widetilde{H}_{\ell+1}\right)=S p\left(H_{\ell}\right) \cup\{\epsilon\}$. In any other case $S p\left(\widetilde{H}_{\ell+1}\right)=S p\left(H_{\ell}\right)$.

In particular, if we chose $\epsilon=-\kappa^{2} \ell^{2}$ and consider (16) and (54), we write

$$
\widetilde{W}_{\ell}(x)=\kappa \lambda \operatorname{sech}^{2}(\kappa u(x))+\ell \widetilde{\beta}(x),
$$

where the function $\widetilde{\beta}$ must satisfy the Riccati equation

$$
-\frac{1}{J} \frac{d \widetilde{\beta}}{d x}+2 \kappa \lambda \operatorname{sech}(\kappa u) \widetilde{\beta}+\ell \widetilde{\beta}^{2}-\kappa^{2} \ell=-\kappa^{2}(\ell-1) \operatorname{sech}^{2}(\kappa u(x))-\kappa^{2} \lambda \operatorname{sech}(\kappa u) \tanh (\kappa u) .
$$

The general solution to this equation reads

$$
\widetilde{\beta}(x)=-\kappa \tanh (\kappa u(x))-\frac{1}{\ell J(x)} \frac{d}{d x} \ln \left[\gamma-\ell \int^{x} \rho(y) J(y) d y\right],
$$

with $\gamma$ an integration constant and

$$
\rho(x)=[\cosh (\kappa u(x))]^{-2 \ell} \exp \{2 \lambda \arctan [\sinh (\kappa u(x))]\} .
$$

The new almost isospectral potential turn out to be

$$
\begin{aligned}
\widetilde{V}_{\ell+1}(x)=V_{\ell+1} & +\frac{2}{J^{2}(x)}\left(\frac{d}{d x} \ln J(x)\right) \frac{d}{d x} \ln \left[\gamma-\ell \int^{x} \rho(y) J(y) d y\right]+ \\
& -\frac{2}{J^{2}(x)} \frac{d^{2}}{d x^{2}} \ln \left[\gamma-\ell \int^{x} \rho(y) J(y) d y\right],
\end{aligned}
$$

where regular potentials of this type are granted for choices of $\gamma$ such that

$$
|\gamma|>\max \left[\ell \int^{x} \rho(y) J(y) d y\right] .
$$

The value of $\epsilon=\epsilon_{\ell}=-\kappa^{2} \ell^{2}$ is not contained in $S p\left(H_{\ell}\right)$, so that we look for a missing state. In this case, we obtain

$$
\widetilde{\psi}_{\ell+1}^{\epsilon}(x)=N_{\ell+1}^{\epsilon} \exp \{\lambda \arctan [\sinh (\kappa u(x))]\} \frac{\cosh ^{-\ell}(\kappa u(x))}{\gamma-\ell \int^{x} \rho(y) J(y) d y} .
$$

As this is a normalizable function in $\operatorname{Dom}\left(\widetilde{V}_{\ell+1}\right)$, it must belong to the complete set of eigenvectors and $S p\left(\widetilde{H}_{\ell+1}\right)=$ $S p\left(H_{\ell}\right) \cup\left\{-\kappa^{2} \ell^{2}\right\}=S p\left(H_{\ell+1}\right)$. 


\section{SUMMARY AND CONCLUSIONS}

We have presented a method of constructing families of PDM Scarf Hamiltonian hierarchies of the trigonometric as well as the hyperbolic type. In this method, it is assumed that the underlying potential or spectrum generating algebra is known and the potential is determined by using a Riccati equation. We have constructed families of PDM Scarf potentials and used the factorization method to find the corresponding eigenfunctions. Some types of the mass function with and without singularities were considered in order to illustrate our results in different situations. Next, we used these Scarf potentials in order to construct new PDM potential with the Scarf spectrum by means of supersymmetric transformations. The convenience of using this approach lies in the fact that we may generate hierarchies of PDM Hamiltonians with a specific discrete spectrum. As the potential algebra is already fixed, the construction of ladder operators, dynamical algebras and coherent states can be directly generalized from the constant mass regime. Additionally, it is worthwhile to mention that our results are consistent for any particular ordering one may chose in order to construct a proper PDM Hamiltonian, as in our model neither the Hamiltonian nor the factorizing operators are dependent of the ordering parameter $a$. This formalism can be extended to potentials of the Pöschl-Teller, Morse and Eckart types, among others. ${ }^{26}$ Results in this direction are in progress and will be reported elsewhere.

\section{ACKNOWLEDGEMENTS}

The authors acknowledge the financial support of CONACyT (PhD scholarship for C. Santiago-Cruz, grant 408518), Instituto Politécnico Nacional, México (Project SIP 20180377), the Spanish MINECO (Project MTM2014-57129-C2-1-P), and Junta de Castilla y León (VA057U16).

\section{ORCID}

S Cruzy Cruz (i) http://orcid.org/0000-0002-7133-0803

\section{REFERENCES}

1. Infeld L, Hull TE. The factorization method. Rev Mod Phys. 1951;23:21-68.

2. Andrianov AA, Borisov NV, Ioffe MV. The factorization method and quantum-systems with equivalent energy-spectra Phys. Lett $A$. 1984;105:19.

3. Mielnik B, Rosas-Ortiz O. Factorization: little or great algorithm. J Phys A: Math Gen. 2004;37:10007-10035.

4. Kuru Ş, Negro J. Dynamical algebras for Pöschl-Teller Hamiltonians hierarchies. Ann Phys. 2009;324:2548-2560.

5. Mielnik B. Factorization method and new potentials with the oscillator spectrum. J Math Phys. 1984;25:387-389.

6. Andrianov AA, Borisov NV, Ioffe MV, Eides MI. Supersymmetric mechanics: a new look at the equivalence of quantum systems. Theor Math Phys. 1984;61:965-972.

7. Sukumar CV. Supersymmetry, factorisation of the Schrödinger equation and a Hamiltonian hierarchy. J Phys A: Math Gen. 1985;18:L57-L61.

8. De R, Dutt R, Sukhatme U. Mapping of shape invariant potentials under point canonical transformations. $J$ Phys A: Math Gen. 1992;25:L843-L850.

9. Cooper F, Khare A, Sukhatme U. Supersymmetry in quantum mechanics. Phys Rep. 1995;251:267-385.

10. Bagchi BK. Supersymmetry in Quantum and Classical Mechanics. London Boca Raton FL: Chapman and Hall CRC Press; 2000.

11. Wannier GH. The structure of electronic excitation levels in insulating crystals. Phys Rev. 1937;52:191-197.

12. von Roos O. Position-dependent effective masses semiconductor theory. Phys Rev B. 1983;27:7547.

13. Khordad R. Hydrogenic donor impurity in a cubic quantum dot: effect of position-dependent effective mass. Europhys $J$ B. $2012 ; 85: 114$.

14. Panahi H, Golshahi S, Doostdar M. Influence of position dependent effective mass on donor binding energy in square and V-shaped quantum wells in the presence of a magnetic field. Phys B. 2013;418:47-51.

15. Cariñena JF, Rañada MF, Santander M. Curvature-dependent formalism, Schrödinger equation and energy levels for the harmonic oscillator on three-dimensional spherical and hyperbolic spaces. J Phys A: Math Theor. 2012;45(265303).

16. Cariñena JF, Rañada MF, Santander M. The quantum free particle on spherical and hyperbolic spaces: a curvature dependent approach. II. J Math Phys. 2012;53(102109).

17. Richstone DO, Potter MD. Galactic mass loss: a mild evolutionary correction to the angular size test. Astrophys J. 1982;254:451-455.

18. Milanović V, Ikonić Z. Generation of isospectral combinations of the potential and the effective-mass variations by supersymmetric quantum mechanics. J Phys A: Math Gen. 1999;32:7001-7015.

19. Plastino AR, Rigo A, Casas M, Gracias F, Plastino A. Supersymmetric approach to quantum systems with position-dependent effective mass. Phys Rev A. 1999;60:4318. 
20. Gönül B, Gönül B, Tutcu D, Özer O. Supersymmetric approach to exactly solvable systems with position-dependent effective mass. Mod Phys Lett A. 2002;17:2057-2066.

21. Koç R, Koca M. A systematic study on the exact solution of the position dependent mass Schrödinger equation. $J$ Phys $A$ Math Gen. 2003;36:8105-8112.

22. Roy B, Roy P. Effective mass Schrödinger equation and nonlinear algebras. Phys Lett A. 2005;340:70.

23. Quesne C. First-order intertwining operators and position-dependent mass Schrödinger equations in d dimensions. Ann Phys. 2006;321:1221.

24. Cruz y Cruz S, Negro J, Nieto LM. Classical and quantum position-dependent mass harmonic oscillators. Phys Lett A. 2007;369:400-406.

25. Bagchi B, Tanaka T. A generalized non-Hermitian oscillator Hamiltonian, N-fold supersymmetry and position-dependent mass models. Phys Lett A. 2008;372:5390-5393.

26. Quesne C. Point canonical transformation versus deformed shape invariance for position-dependent mass Schrödinger equations. SIGMA. 2009;5:046.

27. Cruz y Cruz S, Rosas-Ortiz O. Position-dependent mass oscillators and coherent states. J Phys A: Math Theor. 2009;42(185205).

28. Cruz y Cruz S. Factorization Method and the Position-dependent Mass Problem, Trends in Mathematics (Germany Springer Basel) $229 ; 2013$.

29. Santiago-Cruz C. Isospectral trigonometric Pöschl-Teller potentials with position dependent mass generated by supersymmetry. $J$ Phys Conf Ser. 2016;698:012028.

30. Yahiaoui S-H, Bentaiba M. Isospectral Hamiltonian for position-dependent mass for an arbitrary quantum system and coherent states. J Math Phys. 2017;58:063507.

31. Amir N, Iqbal S. Ladder operators and associated algebra for position-dependent effective mass systems. EPL. 2015;111:20005.

32. Suzko AA, Tralle I. Reconstruction of quantum well potentials via the intertwining operator technique. Acta Phys Pol B. 2008;39:545.

33. Rosas-Ortiz O, Castaños O, Dieter S. New supersymmetry-generated complex potentials with real spectra. $J$ Phys A: Math Theor. 2015;48:445302.

34. Lévy-Leblond JM. Position-dependent effective mass and Galilean invariance. Phys Rev A. 1995;52:1845-1849.

35. Ganguly A, Kuru Ş, Negro J, Nieto LM. A study of the bound states for square potential wells with position-dependent mass. Phys Lett A. 2006;360:228-233.

36. Mustafa O, Mazharimousavi SH. Ordering ambiguity revisited via position-dependent mass pseudo-momentum operators. Int J Theor Phys. 2007;46:1786-1796.

37. Abramowitz M, Stegun I. Handbook of Mathematical Functions with Formulas, Graphs and Mathematical Tables. Washington DC: Dover; 1970.

38. Cruz y Cruz S, Kuru Ş, Negro J. Classical motion and coherent states for Pöschl-Teller potentials. Phys Lett A. 2008;372:1391-1405.

39. Cruz y Cruz S, Negro J, Nieto LM. On the position dependent mass harmonic oscillatos. J Phys Conf Ser. 2008;128(012053).

40. Perelomov A. Generalized Coherent States and Their Applications. Berlin: Springer-Verlag; 1986.

41. Rosas-Ortiz O, Cruz y Cruz S, Enriquez M. SU(1,1) and SU(2) approaches to the radial oscillator: generalized coherent states and squeezing of variances. Ann Phys. 2016;373:346-373.

42. Cruz y Cruz S, Gress Z. Group approach to the paraxial propagation of Hermite-Gaussian modes in a parabolic medium. Ann Phys. 2017;383:257-277.

43. Perstch F. Exact solution of the Schrodinger equation for a potential well with a barrier and other potentials. $J$ Phys A: Math Gen. 1990;23:4145.

44. Zhang X-C, Liu Q-W, Jia C-S, Wang L-Z. Bound states of the Dirac equation with vector and scalar Scarf-type potentials. Phys Lett A. 2005;340:59.

45. Bagchi B, Quesne C. $\operatorname{sl}(2, \mathbb{C})$ as a complex Lie algebra and the associated non-Hermitian Hamiltonians with real eigenvalues. Phys Lett $A$. 2000:285.

46. Bagchi B, Quesne C. Non-hermitian Hamiltonians with real and complex eigenvalues in a Lie-algebraic framework. Phys Lett A. 2002;300:18.

47. Bagchi B, Quesne C. An update on the $\mathcal{P} T$-symmetric complexified Scarf II potential, spectral singularities and some remarks on the rationally extended supersymmetric partners. J Phys A: Math Theor. 2010;43:305301.

How to cite this article: Cruzy Cruz S, Santiago-Cruz C. Position dependent mass Scarf Hamiltonians generated via the Riccati equation. Math Meth Appl Sci. 2018;1-16. https://doi.org/10.1002/mma.5068 